Research Article

\title{
In Silico Analysis of Common Long Noncoding RNAs in Schistosoma mansoni and Schistosoma haematobium
}

\author{
Serhat Sirekbasan (iD) and Tugba Gurkok Tan (iD) \\ Department of Medical Laboratory Techniques, Eldivan Vocational School of Health Services, Çankırı Karatekin University, \\ Çankırı, Turkey \\ Correspondence should be addressed to Serhat Sirekbasan; serhatsirekbasan@gmail.com
}

Received 15 October 2020; Revised 2 February 2021; Accepted 4 February 2021; Published 15 February 2021

Academic Editor: Pedro P. Chieffi

Copyright ( $) 2021$ Serhat Sirekbasan and Tugba Gurkok Tan. This is an open access article distributed under the Creative Commons Attribution License, which permits unrestricted use, distribution, and reproduction in any medium, provided the original work is properly cited.

\begin{abstract}
Background. Schistosomiasis caused by Schistosoma parasites is one of the most common parasitic infections worldwide. Genetic regulation of the genus Schistosoma, which has different developmental stages throughout its life, is quite complex. In these parasites, thousands of long noncoding RNAs (lncRNAs) estimated to be functional were identified. Identifying the transcripts expressed in common and detecting their functions for better understanding of the role of these lncRNAs require a comparative study. Methods. Assembled RNA-seq datasets belonging to S. mansoni and S. haematobium were obtained from the National Center for Biotechnology. A basic local alignment search tool (BLASTN) analysis was conducted against previously constructed lncRNA library to identify the common lncRNAs between two species. LncRNAs target genes and their gene ontology annotation was performed. Results. In S. mansoni and S. haematobium, 5132 and 3589 lncRNA transcripts were detected, respectively. These two species had 694 lncRNAs in common. A significant number of lncRNAs was determined to be transcribed from sex chromosomes. The frequently expressed lncRNAs appear to be involved in metabolic and biological regulation processes. Conclusions. These two species share similar lncRNAs; thus, this finding is a clue that they might have similar functions. In sexual development, they especially might play important roles. Our results will provide important clues to further studies about interactions between human hosts and parasites and the infection mechanisms of Schistosoma parasites.
\end{abstract}

\section{Introduction}

Schistosomiasis caused by Platyhelminthes of the genus Schistosoma is one of the most serious parasitic diseases in humans. It affects more than 240 million people in 78 countries worldwide and causes approximately 200,000 deaths per year [1]. Three main Schistosoma species can infect humans: (1) S. haematobium, (2) S. mansoni, and (3) S. japonicum. S. mansoni and S. japonicum cause intestinal schistosomiasis, whereas $S$. haematobium causes urogenital schistosomiasis $[2,3]$.

In contrast to other trematodes, the Schistosoma genus shows gender differentiation (male and female) [4], which have eight pairs of chromosomes, seven of which are autosomes and one is a pair of sex chromosomes [5]. In the life cycle of the Schistosoma species, intermediate and definite hosts with different developmental stages are found; hence, complex morphological changes in these organisms occur [6].

The elucidation of all these host-parasite relationships depends on increasing our knowledge of the Schistosoma genome, transcriptome, and proteome. The genetic changes or similarities that shape the evolution of the parasite are likely to play a role in host infectivity, development and differentiation, drug susceptibility, pathogenicity, and immunogenicity [6].

With the evolution of animal multicellularity, several regulatory gene expression systems developed, such as transcription factors and noncoding RNAs. Therefore, it is important to explain the regulation of gene expression in these organisms in addition to the many environmental factors required for the formation of schistosomiasis. 
Formerly, the only well-known ncRNAs were the ribosomal RNAs (rRNA), transfer RNAs (tRNA), and small RNAs. Nevertheless, with the advancement of high-throughput sequencing techniques and computational pipelines, new ncRNAs and their functions in genome and gene regulation has been elucidated. One of the newly characterized ncRNAs is the long noncoding RNAs (lncRNAs) lncRNAs. LncRNAs are defined as noncoding transcripts longer than $200 \mathrm{nu}$ cleotides and do not encode an open reading frame (ORF) of more than 100 amino acids [7]. They have important roles in gene expression, developmental and differentiation processes, and genomic imprinting [8, 9]. Besides, lncRNAs interact with other RNA species, such as microRNAs and mRNAs [10].

Classification of lncRNAs is based on several characters, such as their genomic localizations, transcript length, association with annotated protein-coding genes, and/or sequence and structure conservation $[11,12]$. LncRNAs can be placed into several categories: (1) long intergenic noncoding RNAs (lincRNAs), (2) intronic lncRNAs (transcribed within the introns of protein-coding genes), or (3) antisense lncRNAs [8]. It has been reported that despite most proteincoding sequences, lncRNAs rapidly evolve and display poor primary sequence similarity between species [13].

Multicellular organisms include thousands of different lncRNA sequences in their genomes [14]. To date, from protozoans to mammals, a huge number of lncRNAs have been identified [15-18]. Over the past several decades, a number of studies on lncRNAs in Schistosoma species have been conducted $[19,20]$. However, molecular studies of Schistosoma species ncRNAs are limited.

In this study, we aimed to determine the homologybased putative protected lncRNA sequences in S. mansoni and S. haematobium species and to determine their common functions among species according to their target genes.

\section{Materials and Methods}

2.1. Data Sources. RNA-seq datasets were obtained from Transcriptome Shotgun Assembly (TSA) database from National Center for Biotechnology Information (NCBI) under accession numbers GDUI01 (S. mansoni) and GGGJ01 (S. haematobium).

2.2. Homology-Based Identification of $\operatorname{lncRNAs.~Sequence~}$ homology between $S$. mansoni and $S$. haematobium lncRNAs was analyzed using a basic local alignment search tool (BLASTN) [21]. Both S. mansoni and S. haematobium assembled reads were aligned with the $S$. mansoni lncRNA library [20]. The sequences with $>90 \%$ identity and a $p$ value of $10^{-5}$ were predicted to be candidate lncRNAs.

\subsection{Selecting the Transcripts according to Their Coding} Potential. After finding the candidate lncRNA encoding transcripts, we filtered $<200 \mathrm{bp}$, and the remaining transcripts were uploaded to the coding potential calculator (CPC) website (http://cpc.cbi.pku.edu.cn) for coding potential analysis [22]. CPC provides coding probability, isoelectric points, and Fickett scores and contributes a probability as to whether the transcripts are coding or noncoding. Parameters for the website were set to use only the forward strand. All transcripts with CPC scores $>0.5$ were discarded and remaining transcripts were considered as noncoding. Also, candidates were then searched against Pfam protein database (http://pfam.xfam.org/). Noncoding transcripts were used for further analysis.

2.4. Putative Target Gene Prediction and Enrichment Analysis. Previously reported antisense targets of the lncRNAs [20] were detected for the transcripts detected in our experiments. Target genes gene ontology (GO) annotation, including the GO biological process (BP), cellular component (CC), and molecular function (MF), was conducted using Uniprot database [23]. Also, the classification of the lncRNAs was performed according to a previous study [20].

\section{Results}

3.1. Transcriptome-Wide Identification of LncRNAs. In order to explore the lncRNAs, we used egg, adult male, and adult female assembled transcriptome libraries of S. mansoni and S. haematobium. Several steps of the bioinformatics pipeline were used to detect lncRNAs. Transcripts $(23,678$ and 31,591 transcripts) were previously created in S. mansoni [24] and S. haematobium (unpublished data), respectively.

We performed BLASTN to detect the known lncRNAS in S. mansoni and found 28355 hits. After removing the transcripts that were $<200 \mathrm{bp}$, the number of remaining transcripts was 6110 . The transcripts greater than $200 \mathrm{bp}$ were also run through CPC. Of the 6110 sequences analyzed by CPC, 5132 were classified as noncoding. Among 5132 lncRNA transcripts found to be noncoding, we detected 1669 that were unique. Using the previous data created by Vasconcelos et al. [20], our results demonstrated that 213 of them had antisense lncRNAs, whereas 1456 of them were shown to be lincRNAs. We also detected the count numbers of S. mansoni lncRNAs in the RNA-seq library. SmAS00326 (27 read numbers) and SmLINC01644 (24 read numbers) were the most abundant lncRNAs (Table 1). The distribution of the lncRNAs to chromosomes showed that most of the lncRNA encoding genes were located at chromosome 1 and chromosome ZW (Figure 1).

In the results of $S$. haematobium, we detected 4372 transcripts longer than $200 \mathrm{bp}$ from which a total of 3589 were represented as noncoding based on CPC analysis. We also detected 509 lncRNA encoding transcripts that were capable of targeting several S. mansoni mRNAs. All of these belonged to the antisense lncRNAs. The majority of S. haematobium $\operatorname{lncRNAs}$ were found to be intergenic (82\%). Analysis of the abundance of IncRNAs revealed that SmLINC01683 was the most abundant with 27 transcripts (Table 1). Our results revealed that the number of lncRNA transcripts belonging to $S$. mansoni was higher than S. haematobium.

The number of common lncRNAs between S. haematobium and S. mansoni species was 694 (Figure 2). 
TABLE 1: Long noncoding RNA transcripts showing high read numbers in S. mansoni and S. haematobium.

\begin{tabular}{lccc}
\hline LncRNA & Read number & Target gene & Annotation \\
\hline S. mansoni & & & Unidentified \\
SmAS00026-IBu & 27 & Unidentified & Unidentified \\
SmLINC01664-IBu & 24 & Unidentified & GTPase activity \\
SmAS00327-IBu & 20 & Smp_169460 (Rab, putative) & Unidentified \\
SmLINC01664-IBu & 16 & Unidentified & Unidentified \\
SmAS00186-IBu & 15 & Uncharacterized protein & Unidentified \\
S. haematobium & & & Unidentified \\
SmAS00186-Ibu & 26 & Uncharacterized protein & Unidentified \\
SmAS00167-Ibu & 20 & Unidentified & Unidentified \\
SmAS00326-Ibu & 19 & Unidentified & Unidentified \\
SmAS00328-Ibu & 19 & Unidentified & Unidentified \\
SmAS00041-Ibu & 16 & Unidentified & Unidentified \\
SmAS00042-Ibu & 16 & Unidentified & Unidentified \\
SmAS00043-Ibu & 16 & Unidentified & Unidentified \\
SmAS00044-Ibu & 16 & Unidentified & Unidentified \\
SmAS00045-Ibu & 16 & Smp_169460 (uncharacterized & compound organization \\
SmAS00178-Ibu & 16 & & \\
\hline
\end{tabular}

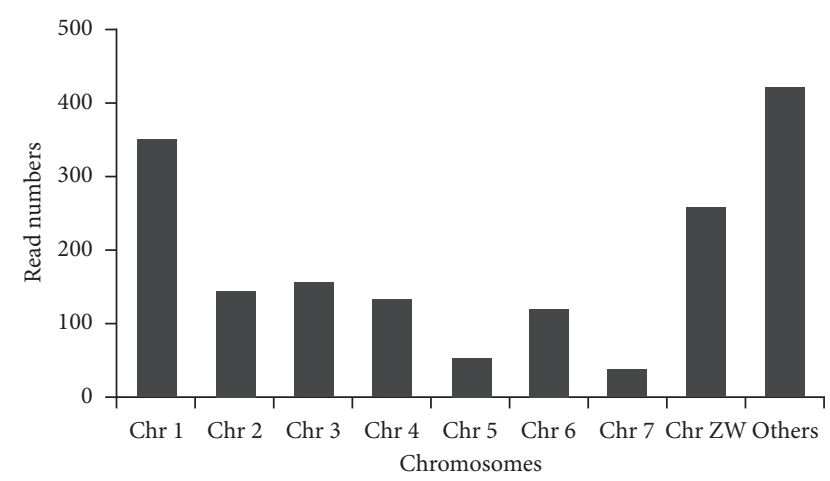

FIGURE 1: Location of the long noncoding RNA (lncRNA) transcripts and their read numbers in S. mansoni chromosomes. Others, lncRNAs that have not been mapped to any chromosomes.

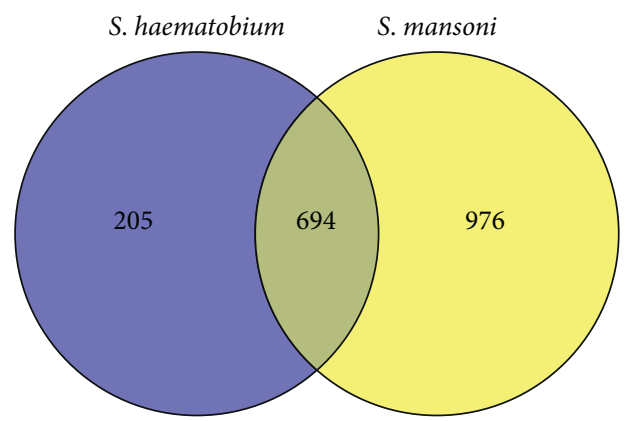

FIGURE 2: Venn diagram demonstrating shared and unique lncRNA transcripts.

The most abundant type was lincRNAs with the rate of $85 \%$. A large number of their target annotations were found to be uncharacterized. SmAS00186 and SmAS00326 were represented by the highest number of common transcripts.

3.2. Target GO Enrichment Analyses. To identify and annotate the GO categories of the target genes in both
S. mansoni and S. haematobium, we conducted GO enrichment analyses in terms of biological processes, molecular functions, and cellular components. The identification was carried out using UniProt.

In S. mansoni, only $10 \%$ of the transcripts had target genes. In biological processes, the majority of the target genes existed in relation to biological regulation $(31 \%)$ and metabolic processes (24\%) as shown in Figure 3. The GO analysis revealed binding of $46 \%$ in relation to molecular function and $23 \%$ in relation to integral membrane components in cellular compartments.

In S. haematobium, protein metabolic processes (26\%) and metabolic processes (21\%) showed a consistently higher number of target genes. Also, the results indicate that the largest group of transcripts belongs to binding (38\%) and protein-containing complexes (43\%) related to molecular functions and cellular processes (Figure 4).

Among common target genes, the highest number of transcripts was involved in the metabolic process (26\%) and cellular component organization (24\%) in biological processes. The transcripts related to binding and integral membrane components had a significant number of target genes, respectively (Figure 5).

\section{Discussion}

Studying regulatory ncRNAs, such as miRNAs or lncRNAs, provides important clues about gene expression and/or evolutionary mechanisms. High-throughput next-generation sequencing has brought about the discovery of thousands of noncoding RNA genes (ncRNAs), such as short, long, and circular RNAs, over the last several decades. Also, several studies have been conducted for identification of both miRNAs and IncRNAs in Schistosoma genomes [25-27].

Schistosomiasis is a worldwide disease with insufficient data on its developmental processes and disease-inducing processes. Schistosomiasis is a worldwide disease with insufficient data on the molecular mechanisms of host-parasite 


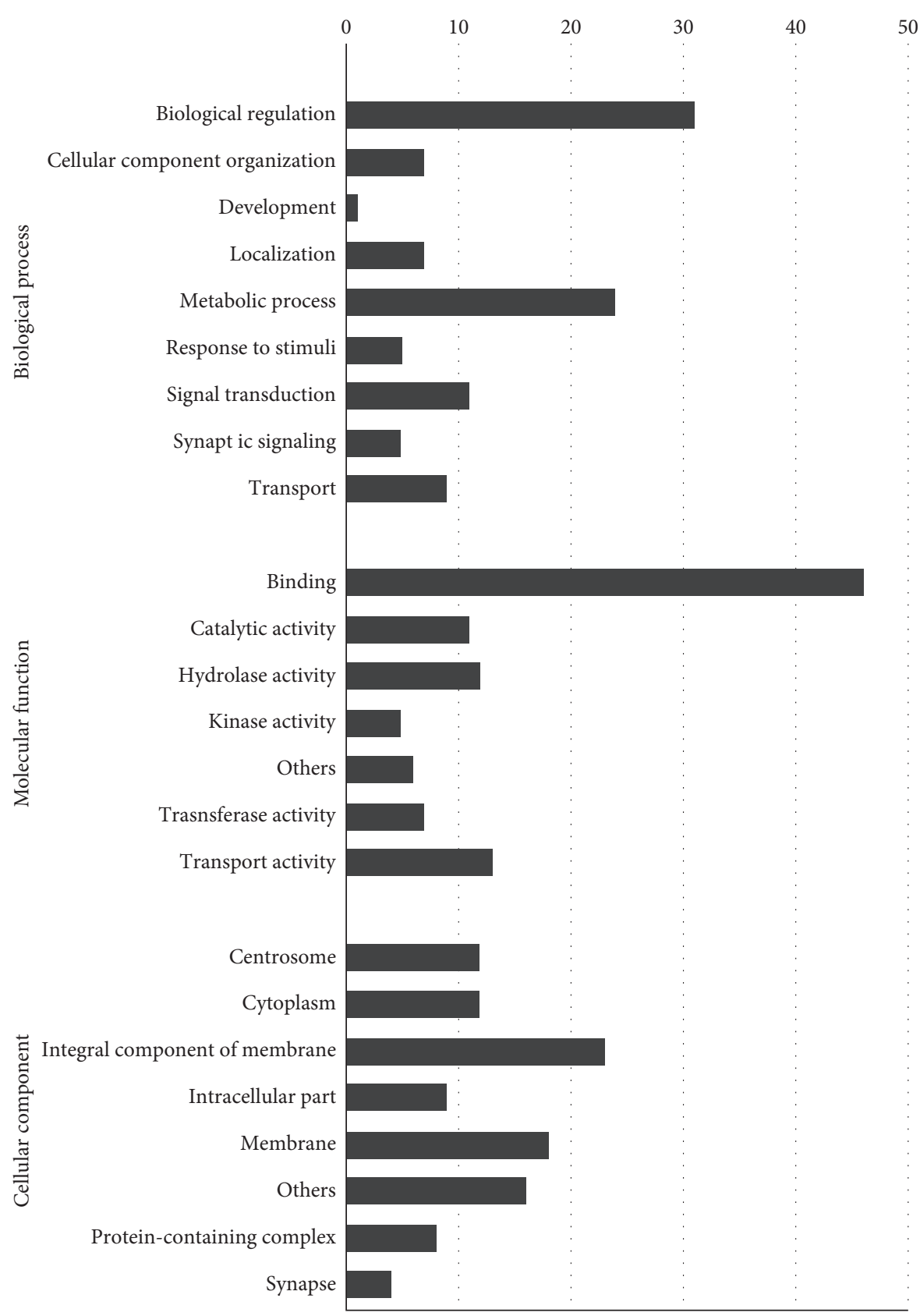

Figure 3: Percentage representation of gene ontology (GO) enrichment analysis of lncRNA targets in S. mansoni.

interaction and the epigenetic changes involved in this process. Little is known about the genes and molecules that direct the biological process in the complex life cycle of the disease-causing parasite that includes different developmental stages. In this study, we aimed to determine the common homology-based IncRNAs between the two species using the comparative transcriptomic approach in both species adult male, female, and eggs, if there were any similar regulatory systems between these two species.

We detected 1669 and $899 \operatorname{lncRNAs}$ of S. mansoni and S. haematobium. Besides, we found significant number of transcripts that were expressed in both S. mansoni and S. haematobium transcriptome libraries. In agreement with our findings, several studies indicate that a higher species similarity between S. mansoni and S. haematobium in the case of lncRNAs and mRNAs exists [19]. Although we did not search the mRNA sequence similarity, BLASTN results give us a general insight about the lncRNA sequence similarity between these two species. Indeed, it has been reported that lncRNAs sequence conservation is lower than mRNA [17], and a reduction in levels of primary sequence conservation for lncRNAs among different organisms during evolution was found [13]. In this study, we detected a prominent number of similar lncRNAs between two species in egg, adult male, and adult female libraries. Previously, it was noticed that transcription of lncRNAs appears to be more active in the early embryonic stage [28]. This finding indicates that in early developmental stages, the same 


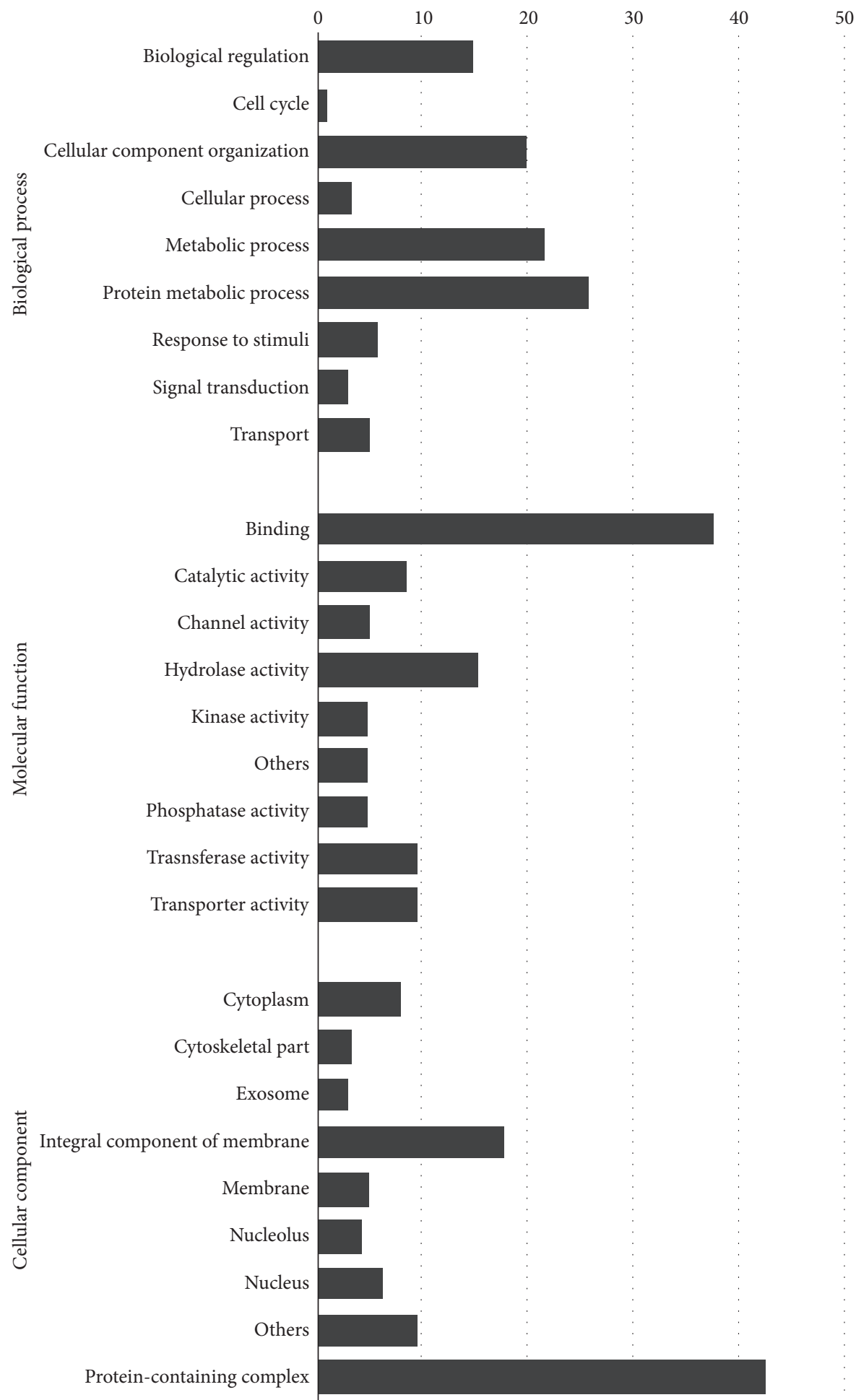

FIgURe 4: Percentage representation of GO enrichment analysis of lncRNA targets in S. haematobium.

lncRNAs show parallel functions. In addition to a role in regulation of developmental gene activity, the role of lncRNAs was found to be widespread amongst metazoans $[29,30]$.

Different studies have revealed that lncRNAs play important roles in sexual development and determination in eukaryotes $[31,32]$. In this study, a significant number of transcripts encoding lncRNAs located on chromosome 1 and sex chromosomes in S. mansoni were detected (Figure 1). In parallel, Oliveira et al. [26] reported in their research that the significant number of lncRNAs was found on chromosome 1 , sexual ZW. It was indicated that there was a gene dosage differentiation in Schistosoma species [33]. Hence, we can argue about the functions of lncRNAs in sexual development. 


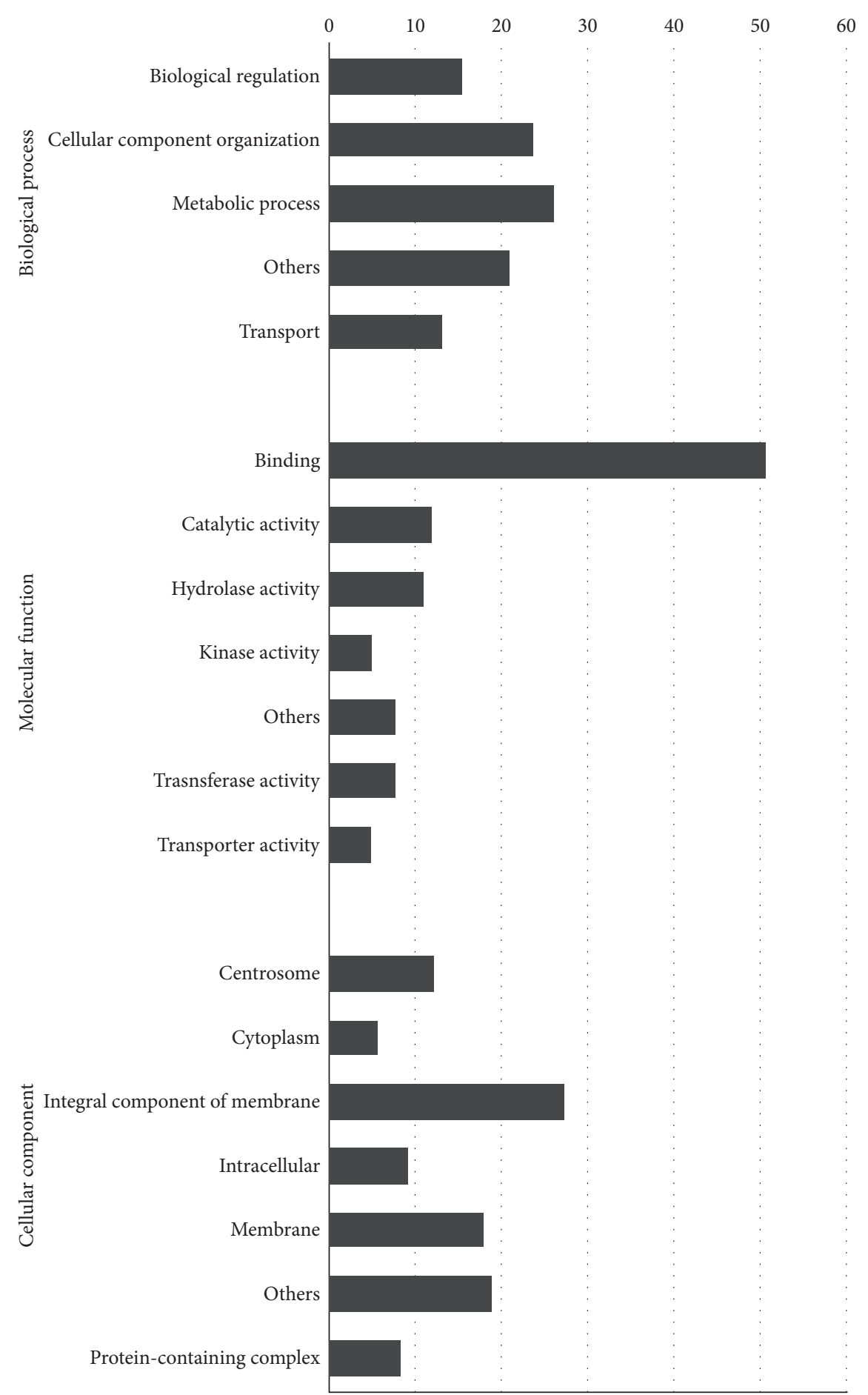

FIGURE 5: Percentage representation of GO enrichment analysis of shared lncRNA targets in S. mansoni and S. haematobium.

Since lncRNA targets may consist of more than one target, they can influence gene expression in many biological processes [34]. In our study, some differences between $S$. mansoni and S. haematobium biological processes, molecular functions, and cellular compartments were found. A large number of target genes associated with the metabolic process have been identified in both $S$. mansoni and S. haematobium. On the other hand, while genes related to biological regulation were determined in large numbers in
S. mansoni, genes related to protein metabolic function were found in large numbers in S. haematobium. In the study of Oliveira et al. [26], it was stated that most of the lncRNAs identified in S. mansoni were related to metabolism. As indicated before, these species were located in different tissues because their gene expression is regulated by different lncRNAs. Besides, parasite lncRNAs can play crucial roles in a tissue-specific manner in humans. It was shown by Maciel et al. [35] that some lncRNAs in S. mansoni have a tissue- 
specific expression in ovaries and testis. In this study, the authors obtained valuable information on the potential role of $\operatorname{lncRNAs}$ in schistosome reproduction. However, lncRNAs may be involved in a specific role related to the tissue in which they are located. Also, lncRNA gene expression can vary in different developmental stages in parasites; so, it is possible for these molecules to play an active role in the early diagnosis and treatment of the schistosomiasis. Although it is largely unknown whether lncRNA levels can be regulated by drugs, some studies have shown the effect of drugs on lncRNA expression [36-38]. A better understanding of lncRNAs and gene expression regulation mechanisms can help identify new therapeutic targets.

It has been reported that $\operatorname{lncRNAs}$ can be speciesspecific or even tissue-specific and that the sequence similarity organisms may be conserved in the evolutionary process [8]. Also, it was claimed that highly conserved sequences may be related to the functionality of lncRNAs among organisms [25]. However, we could not fully perform functional annotation for lncRNAs of schistosome due to minimal functional information of known genes and the limited number of RNA-seq samples. We found that $S$. mansoni and S. haematobium IncRNA sequences are conserved with $7.9 \%$ of the S. haematobium IncRNAs in alignment with the S. mansoni transcriptome.

\section{Conclusions}

In this study, we used the S. mansoni lncRNA library, so this process may explain why we detected more lncRNAs in S. mansoni than in S. haematobium. In addition, it is thought that lncRNAs, which are commonly expressed in accordance with the resulting data, are involved in both metabolic and biological regulation functions.

\section{Data Availability}

The data used to support the findings of this study are available from the corresponding author upon request.

\section{Conflicts of Interest}

The authors declare that there are no conflicts of interest.

\section{Authors' Contributions}

SS and TGT designed the model and the computational framework and analyzed the data. SS carried out the implementation. TGT performed the calculations. Both the authors discussed the results and contributed to the final manuscript.

\section{References}

[1] World Health Organization (WHO), "Schistosomiasis: number of people treated worldwide in 2014," Releve Epidemiologique Hebdomadaire, vol. 91, no. 5, pp. 53-60, 2016.
[2] D. G. Colley, A. L. Bustinduy, W. E. Secor, and C. H. King, "Human schistosomiasis," The Lancet, vol. 383, no. 9936, pp. 2253-2264, 2014.

[3] D. P. McManus, D. W. Dunne, M. Sacko, J. Utzinger, B. J. Vennervald, and X. N. Zhou, "Schistosomiasis," Nature Reviews Disease Primers, vol. 4, no. 1, p. 13, 2018.

[4] K. F. Hoffmann, D. A. Johnston, and D. W. Dunne, "Identification of Schistosoma mansoni gender-associated gene transcripts by cDNA microarray profiling," Genome Biology, vol. 3, no. 8, 2002.

[5] Y. Hong, Z. Fu, X. Cao, and J. Lin, "Changes in microRNA expression in response to Schistosoma japonicum infection," Parasite Immunology, vol. 39, no. 2, Article ID e12416, 2017.

[6] Z.-G. Han, P. J. Brindley, S.-Y. Wang, and Z. Chen, "Schistosoma genomics: new perspectives on schistosome biology and host-parasite interaction," Annual Review of Genomics and Human Genetics, vol. 10, no. 1, pp. 211-240, 2009.

[7] P. Kapranov, J. Cheng, S. Dike et al., "RNA maps reveal new RNA classes and a possible function for pervasive transcription," Science, vol. 316, no. 5830, pp. 1484-1488, 2007.

[8] C. P. Ponting, P. L. Oliver, and W. Reik, "Evolution and functions of long noncoding RNAs," Cell, vol. 136, no. 4, pp. 629-641, 2009.

[9] A. Tichon, N. Gil, Y. Lubelsky et al., "A conserved abundant cytoplasmic long noncoding RNA modulates repression by Pumilio proteins in human cells," Nature Communications, vol. 7, p. 12209, 2016.

[10] S. Jalali, D. Bhartiya, M. K. Lalwani, S. Sivasubbu, and V. Scaria, "Systematic transcriptome wide analysis of lncRNA-miRNA interactions," PLoS One, vol. 8, no. 2, Article ID e53823, 2013.

[11] L. Ma, V. B. Bajic, and Z. Zhang, "On the classification of long non-coding RNAs,” RNA Biology, vol. 10, no. 6, pp. 924-933, 2013.

[12] G. St. Laurent, C. Wahlestedt, and P. Kapranov, "The landscape of long noncoding RNA classification," Trends in Genetics, vol. 31, no. 5, pp. 239-251, 2015.

[13] I. Ulitsky, "Evolution to the rescue: using comparative genomics to understand long non-coding RNAs," Nature Reviews Genetics, vol. 17, no. 10, pp. 601-614, 2016.

[14] J. Ponjavic, C. P. Ponting, and G. Lunter, "Functionality or transcriptional noise? Evidence for selection within long noncoding RNAs," Genome Research, vol. 17, no. 5, pp. 556-565, 2007.

[15] M. E. Donaldson and B. J. Saville, "Natural antisense transcripts in fungi," Molecular Microbiology, vol. 85, no. 3, pp. 405-417, 2012.

[16] K. Kim, "The epigenome, cell cycle, and development in toxoplasma," Annual Review of Microbiology, vol. 72, no. 1, pp. 479-499, 2018.

[17] C. K. Chen, C. P. Yu, S. C. Li et al., "Identification and evolutionary analysis of long non-coding RNAs in zebra finch," BMC Genomics, vol. 18, no. 1, p. 117, 2017.

[18] A. M. Khalil, M. Guttman, M. Huarte et al., "Many human large intergenic noncoding RNAs associate with chromatinmodifying complexes and affect gene expression," Proceedings of the National Academy of Sciences, vol. 106, no. 28, pp. 11667-11672, 2009.

[19] Q. Liao, Y. Zhang, Y. Zhu et al., "Identification of long noncoding RNAs in Schistosoma mansoni and Schistosoma japonicum," Experimental Parasitology, vol. 191, pp. 82-87, 2018.

[20] E. J. R. Vasconcelos, L. F. daSilva, D. S. Pires et al., "The Schistosoma mansoni genome encodes thousands of long 
non-coding RNAs predicted to be functional at different parasite life-cycle stages," Scientific Reports, vol. 7, no. 1, p. 10508, 2017.

[21] C. Camacho, G. Coulouris, V. Avagyan et al., "BLAST+: architecture and applications," BMC Bioinformatics, vol. 10, no. 1, p. 421, 2009.

[22] L. Kong, Y. Zhang, Z.-Q. Ye et al., "CPC: assess the proteincoding potential of transcripts using sequence features and support vector machine," Nucleic Acids Research, vol. 35, no. 2, pp. W345-W349, 2007.

[23] The UniProt Consortium, "UniProt: the universal protein knowledgebase," Nucleic Acids Research, vol. 42, pp. D191D198, 2014.

[24] L. Anderson, M. S. Amaral, F. Beckedorff et al., "Schistosoma mansoni egg, adult male and female comparative gene expression analysis and identification of novel genes by RNASeq," PLoS Neglected Tropical Diseases, vol. 9, no. 12, Article ID e0004334, 2015.

[25] M. C. Simões, J. Lee, A. Djikeng et al., "Identification of Schistosoma mansoni microRNAs," BMC Genomics, vol. 12, p. 47, 2011.

[26] V. F. Oliveira, L. A. G. Moares, E. A. Mota et al., "Identification of 170 new long noncoding RNAs in Schistosoma mansoni," Biomed Research International, vol. 2018, Article ID 1264697, 9 pages, 2018.

[27] E. J. R. Vasconcelos, V. C. Mesel, L. F. daSilva et al., "Atlas of Schistosoma mansoni long non-coding RNAs and their expression correlation to protein-coding genes," Database, vol. 2018, Article ID bay068, 2018.

[28] F. Gaiti, S. L. Fernandez-Valverde, N. Nakanishi et al., "Dynamic and widespread lncRNA expression in a sponge and the origin of animal complexity," Molecular Biology and Evolution, vol. 32, no. 9, pp. 2367-2382, 2015.

[29] R. B.-T. Perry and I. Ulitsky, "The functions of long noncoding RNAs in development and stem cells," Development, vol. 143, no. 21, pp. 3882-3894, 2016.

[30] F. Gaiti, A. D. Calcino, M. Tanurdžić, and B. M. Degnan, "Origin and evolution of the metazoan non-coding regulatory genome," Developmental Biology, vol. 427, no. 2, pp. 193-202, 2017.

[31] W. Kim, C. Miguel-Rojas, J. Wang, J. P. Townsend, and F. Trail, "Developmental dynamics of long noncoding RNA expression during sexual fruiting body formation in Fusarium graminearum,” MBio, vol. 9, no. 4, pp. e01292-18, 2018.

[32] A. A. Golicz, P. L. Bhalla, and M. B. Singh, "LncRNAs in plant and animal sexual reproduction," Trends in Plant Science, vol. 23, no. 3, pp. 195-205, 2018.

[33] M. A. L. Picard, C. Cosseau, S. Ferré et al., "Evolution of gene dosage on the Z-chromosome of schistosome parasites," Elife, vol. 7, Article ID e35684, 2018.

[34] P. Avner and E. Heard, "X-chromosome inactivation: counting, choice and initiation," Nature Reviews Genetics, vol. 2, no. 1, pp. 59-67, 2001.

[35] L. F. Maciel, D. A. Morales-Vicente, G. O. Silveira et al., "Weighted gene co-expression analyses point to long noncoding RNA hub genes at different schistosoma mansoni lifecycle stages," Frontiers in Genetics, vol. 10, p. 823, 2019.

[36] K. K. Geyer, C. M. Rodríguez López, I. W. Chalmers et al., "Cytosine methylation regulates oviposition in the pathogenic blood fluke Schistosoma mansoni," Nature Communications, vol. 2, p. 424, 2011.

[37] K. K. Geyer, S. E. Munshi, M. Vickers et al., "The anti-fecundity effect of 5-azacytidine (5-AzaC) on Schistosoma mansoni is linked to dis-regulated transcription, translation and stem cell activities," International Journal for Parasitology: Drugs and Drug Resistance, vol. 8, no. 2, pp. 213-222, 2018.

[38] M. S. Amaral, L. F. Maciel, G. O. Silveira et al., "Long noncoding RNA levels can be modulated by 5-azacytidine in Schistosoma mansoni," Scientific Reports, vol. 10, no. 1, p. $21565,2020$. 